\title{
Increased Prevalence of the IL-6 -174C Genetic Polymorphism in Long Distance Swimmers
}

\author{
by \\ Sigal Ben-Zaken ${ }^{1}$, Yoav Meckel ${ }^{1}$, Dan Nemet ${ }^{2}$,Eias Kassem³ ${ }^{3}$, Alon Eliakim²
}

The IL-6 -174G/C single nucleotide polymorphism (SNP) functionally affects IL-6 activity, with the G-allele associated with increased IL-6 levels. The C-allele was found to be associated with exercise-induced skeletal muscle damage. The aim of the present study was to examine the association between the IL-6 -174G/C polymorphism and athletic performance among elite swimmers and runners. The study sample included 180 track and field athletes and 80 swimmers. Track and field athletes were assigned to three sub-groups: long-distance runners, middle-distance runners and short-distance runners. Swimmers were assigned to two subgroups: long-distance swimmers and short-distance swimmers. The control group consisted of 123 non-athletic healthy individuals. Genomic DNA was extracted from peripheral blood following a standard protocol. Genotyping was performed using polymerase chain reaction (PCR). The CC genotype and C-allele frequency were significantly higher in the long-distance swimmers (18 and 43\%, respectively) compared to the long-distance runners (3 and 14\%, respectively, $p<0.001$ ); middle-distance runners (4 and $22 \%$, respectively, $p<0.001$ ); and controls (5 and 19\%, respectively, $p<0.001$ ). In addition, the CC genotype and $C$-allele frequency were significantly higher $(p<0.001)$ in long-distance swimmers compared to short-distance swimmers (18 versus 5\% and 43 versus 29\% for the CC genotype and C-allele frequency, respectively). The higher frequency of the C-allele and CC genotype among long-distance swimmers suggests that the rarity of exercise-associated rhabdomyolysis among swimmers is probably related to other sports-specific or water-related protective mechanisms. It is possible that swimming selection in talented endurance athletes who are C-allele carriers represents an example of genetically-dependent sports selection.

Key words: IL-6, genetic polymorphism, swimmers, track and field athletes.

\section{Introduction}

Attempts to identify relevant genes for athletic excellence are complicated, because each gene makes only a small contribution to overall heritability. These attempts are particularly important at young age. Children are encouraged to participate in sports at a level consistent with their abilities. Therefore, directing children to exercise at or above their limits, or to specialize in a single sport before adolescence, should be discouraged. Despite this, an increasing number of children do specialize in a specific sport at an early age and compete at the "elite" level. Moreover, selection processes attempt to identify future Olympic champions even before elementary school in certain sports (Valovich McLeod et al., 2011). To make it even more complicated, sports-talented pre-pubertal children often excel in more than one sport discipline, including aerobic and anaerobic, individual or team sports (Gonçalves et al., 2012). Thus, it seems

\footnotetext{
1 - The Zinman College of Physical Education and Sports Sciences at the Wingate Institute, Genetics and Molecular Biology Laboratory, Israel.

2- Meir Medical Center, Child Health and Sports Center, Pediatric Department, Sackler School of Medicine, Tel-Aviv University, Israel.

3. Hilel-Yaffe Medical Center, Pediatric Department, Hadera, Israel.
} 
that a key factor for competitive sports success is the identification of a sport event that best matches the athlete's ability at the proper age. Of the parameters available for possible talent identification, several may be considered as inherited traits. These traits include obviously aspects of body size and proportions, maturity onset and the type of muscle fiber (e.g. fast or slow). Several genetic polymorphisms and profiles have been suggested to promote athletic excellence in endurance (Ben-Zaken et al., 2013; Ruiz et al, 2009; Williams and Folland, 2008) and power sports (Ben-Zaken et al., 2013; Ruiz et al., 2010). However, whether genetic polymorphisms that affect the athlete's ability to tolerate training loads effectively also play a role in sports selection is currently unknown.

Interleukin-6 (IL-6) is a multi-functional cytokine with a wide range of biological activities such as regulation of immune responses, acute phase reactions and erythropoiesis (Hirano, 1998). In addition, IL-6 plays a pivotal role in the processes of muscle hypertrophy and repair following exercise-related muscle damage (Jeunemaitre et al., 1992). Exercise can induce increases in muscle derived IL-6 mRNA and subsequent elevations in circulating IL-6 (Pedersen and Febbraio, 2008; Petersen and Pedersen, 2005). The elevation of circulating IL-6 concentration following exercise is related to exercise intensity and duration, the mass of recruited muscles and endurance capacity (Petersen and Pedersen, 2005).

Studies have shown that the IL-6 -174G/C (rs1800795) single nucleotide polymorphism (SNP) functionally affects IL-6 promoter activity with the $G$ allele associated with increased IL-6 levels (Bennermo et al., 2004; Fishman et al., 1998; Terry et al., 2000). Moreover, the GG genotype and $\mathrm{G}$ allele were found to be over-represented in Spanish (Ruiz et al., 2010), but not Israeli (Eynon et al., 2011), elite power athletes. In contrast, the $C$ allele was found to be associated with eccentric exercise-induced skeletal muscle damage and rhabdomyolysis (Funghetto et al., 2013; Yamin et al., 2008). Interestingly, the prevalence of this SNP has not been studied in swimming, a sport in which fitness and muscle strength (Bloomfield et al., 1985) play a key role in elite performance, but exercise-induced rhabdomyolysis is very rare (Galvez et al., 2008; Stella and Shariff, 2012).
Therefore, the aim of the present study was to examine the association between the IL- 6 174G/C polymorphism and athletic performance among elite Israeli swimmers compared to elite Israeli track and field athletes. While in both sport disciplines increased muscle mass and strength constitute a competitive advantage (mainly in the sprints), exercise-induced muscle damage and rhabdomyolysis are very rare among swimmers. Therefore, we hypothesized that a significantly lower frequency of the IL-6 -174C polymorphism would be found among swimmers.

\section{Material and Methods}

\section{Participants}

One hundred and eighty track and field athletes (138 males and 42 females, age 18-80) and 80 swimmers (49 males and 31 females, age 16-49) participated in the study. Track and field athletes were assigned to three main sub-groups according to their event specialty during their athletic career: 1) long-distance runners (major event: a $5000 \mathrm{~m}$ to marathon run, $\mathrm{n}=63), 2$ ) middle-distance runners (major event: a 800-1500 $\mathrm{m}$ run, $\mathrm{n}=48$ ), and 3) short-distance runners (100$200 \mathrm{~m}$ sprinters and jumpers, $\mathrm{n}=69$ ). Swimmers were assigned to two groups according to their main swimming event during their swimming career: 1) long-distance swimmers (major event: a 400-1500 m swim, $\mathrm{n}=38$ ), and 2) short-distance swimmers (major event: a 50-100 m swim, $\mathrm{n}=42$ ). All athletes were ranked among the top all-time Israeli results in their event and had competed at national and/or international level on a regular basis. The control group consisted of 123 (75 males and 48 females, age 20-29) non-athletic healthy individuals who were not engaged in competitive sport. Characteristics of the athletes and controls are presented in Table 1.

The study was approved by the Institutional Review Board of the Hillel Yaffe Medical Center, Hadera, Israel, according to the Declaration of Helsinki. Written informed consent was obtained from each participant before the commencement of the study.

\section{Genotyping}

Genomic DNA was extracted from peripheral EDTA treated anti-coagulated blood using a standard protocol, described elsewhere (Miller et al., 1988). Briefly, Lyses buffer (5 mL) containing $100 \mathrm{mM}$ of tris $\mathrm{HCl}, \mathrm{pH}=8.5+0.5 \mathrm{M}$ of 
EDTA $+10 \%$ SDS $+5 \mathrm{M}$ of $\mathrm{NaCl} 40 \mathrm{~mL}$ added D.D.W. to $1000 \mathrm{~mL}$ was added to the white cell solution. Proteinase K $(200 \mu \mathrm{g})$ was added to the solution. The tubes were incubated at $50^{\circ} \mathrm{C}$ in a shaker incubator for overnight for digestion. After digestion, one volume of ethanol $\left(-20^{\circ} \mathrm{C}\right)$ was added to the lysate and the samples were swirled until precipitation was completed (20-30 min, until it became completely transparent). The DNA was recovered by lifting the aggregated precipitate from the solution, using a disposable tip. Excess liquid was dabbed off and the DNA was dispersed in a pre-labeled eppendorf tube. The DNA was washed in TE solution. Genotypes were determined using the TaqMan allelic discrimination assay. The Assay-by-Design service (http://www.thermofisher.com) was used to set up a TaqMan allelic discrimination assay for the IL-6 -174G/C (rs1800795). Primer sequences were forward:

\section{GACGACCTAAGCTGCACTTTTC,} reverse:

\section{GGGCTGATTGGAAACCTTATTAAGATTG.}

Probe sequences were for $-174 \mathrm{G} / \mathrm{C}$ forward: VICCTTTAGCAT-GGCAAGAC, reverse: FAMCTTTAGCA TCGCAAGAC. The PCR reaction mixture included $5 \mathrm{ng}$ genomic DNA, $0.125 \mu \mathrm{l}$ TaqMan assay $\left(40^{*}, \mathrm{ABI}\right), 2.5 \mu \mathrm{l}$ Master mix (ABI) and $2.375 \mu \mathrm{l}$ water. PCR was performed in 96 well PCR plates in an ABI 7300 PCR system (Applied Biosystems Inc., Foster City, CA, USA) and consisted of initial denaturation for $5 \mathrm{~min}$ at $95^{\circ} \mathrm{C}$, and 40 cycles with denaturation of $15 \mathrm{~s}$ at $95^{\circ} \mathrm{C}$ and annealing and extension for $60 \mathrm{~s}$ at $63^{\circ} \mathrm{C}$. Results were analyzed by the ABI TaqMan $7900 \mathrm{HT}$ using the sequence detection system 2.22 software (Applied Biosystems Inc.).

\section{Statistical analysis}

The SPSS statistical package, version 20.0, was used to perform all statistical analyses (SPSS, Chicago, IL, USA). A $\chi^{2}$-test was applied to confirm that the observed genotype frequencies were within the Hardy-Weinberg equilibrium and to compare allele and genotype frequencies between athletes and controls, as well as between athletes from different sports and between different competitive groups (e.g., short versus long-distance). If observed or expected values included a cell with a value of 5 , we used a Fisher's exact test to compare allele and genotype frequencies.

\section{Results}

The complete data on allele and genotype frequencies are presented in Table 2. The genotype subtype did not differ by age or sex. The IL-6 genotype distribution was in agreement with the Hardy-Weinberg equilibrium in the longdistance (endurance) athletes $(p=0.74)$, shortdistance (power) athletes $(p=0.61)$ and the control group $(p=0.06)$.

\begin{tabular}{|c|c|c|c|c|c|}
\hline \multicolumn{5}{|c|}{ Athletes and controls' data } & Table 1 \\
\hline Group & $\mathrm{n}$ & Main event & $\mathbf{M} / \mathbf{F}$ & $\begin{array}{l}\text { Top/Natio } \\
\text { nal level }\end{array}$ & $\begin{array}{l}\text { Age } \\
\text { (Mean } \pm S D, \text { range) }\end{array}$ \\
\hline \multicolumn{6}{|l|}{ Runners } \\
\hline Long distance & 63 & $\begin{array}{l}5000 \mathrm{~m}- \\
\text { marathon }\end{array}$ & $51 / 12$ & $22 / 41$ & $32.4 \pm 9.5(17-55)$ \\
\hline Middle distance & 50 & $800-1500 \mathrm{~m}$ & $41 / 9$ & $23 / 27$ & $32.1 \pm 15.0(17-80)$ \\
\hline Short distance & 72 & $\begin{array}{l}\text { 100-200 m, } \\
\text { jumps }\end{array}$ & $49 / 23$ & $23 / 49$ & $30.5 \pm 13.7(17-71)$ \\
\hline \multicolumn{6}{|l|}{$\underline{\text { Swimmers }}$} \\
\hline Long distance & 38 & $400-1500 \mathrm{~m}$ & $22 / 16$ & $9 / 29$ & $25.3 \pm 9.3(16-48)$ \\
\hline Short distance & 42 & $50-100 \mathrm{~m}$ & $27 / 15$ & $8 / 34$ & $23.1 \pm 7.6(16-49)$ \\
\hline Controls & $\begin{array}{l}12 \\
7\end{array}$ & $\mathrm{nr}$ & $77 / 50$ & $\mathrm{nr}$ & $26.4 \pm 5.8(19-29)$ \\
\hline
\end{tabular}


Table 2

The IL-6-174G/C genotype and allele frequencies in all groups. Values are absolute (relative frequencies in parentheses).

\begin{tabular}{|c|c|c|c|c|c|c|c|}
\hline \multirow[b]{2}{*}{ Group } & \multirow[b]{2}{*}{$\mathrm{n}$} & \multicolumn{4}{|c|}{ Genotype frequency } & \multicolumn{2}{|c|}{ Allele frequency } \\
\hline & & $\mathrm{G} / \mathrm{G}$ & $\mathrm{G} / \mathrm{C}$ & $\mathrm{C} / \mathrm{C}$ & $\begin{array}{l}\text { C-allele } \\
\text { carriers }\end{array}$ & G - allele & C - allele \\
\hline Controls & 127 & $84(66)$ & $37(29)$ & $6(5)^{1}$ & $43(34)^{2}$ & $205(81)$ & $49(19)^{3}$ \\
\hline \multicolumn{8}{|l|}{$\underline{\text { Runners }}$} \\
\hline Long distance & 63 & $47(75)$ & $14(22)$ & $2(3)$ & $16(25)$ & $108(86)$ & $18(14)$ \\
\hline Middle distance & 50 & $30(60)$ & $18(36)$ & $2(4)$ & $20(40)$ & $78(78)$ & $22(22)$ \\
\hline Short distance & 72 & $45(63)$ & $26(36)$ & $1(1)$ & $27(37)$ & $116(81)$ & $28(19)$ \\
\hline Total & 185 & $122(66)$ & $58(31)$ & $5(3)^{4}$ & $63(34)^{5}$ & $302(82)$ & $68(18)^{6}$ \\
\hline \multicolumn{8}{|l|}{$\underline{\text { Swimmers }}$} \\
\hline Long distance & 38 & $12(32)$ & $19(50)$ & $7(18)^{7}$ & $26(68)^{8}$ & $43(57)$ & $33(43)^{9}$ \\
\hline Short distance & 42 & $20(48)$ & $20(48)$ & $2(4)$ & $22(52)$ & $60(71)$ & $24(29)$ \\
\hline Total & 80 & $32(40)$ & $39(49)$ & $9(11)$ & $48(60)$ & $103(64)$ & $57(36)$ \\
\hline
\end{tabular}

${ }^{1} \chi 2=14.01, d . f .=2, p<0.001$ for genotype frequency in controls vs. swimmers

${ }^{2} \chi 2=13.62, d . f .=1, p<0.001$ for $C$-allele carriers frequency in controls vs. swimmers ${ }^{3} \chi 2=13.75, d . f .=1, p<0.001$ for allele frequency controls vs. swimmers

${ }^{4} \chi 2=18.81$, d.f. $=2, p<0.001$ for genotype frequency in runners vs. swimmers ${ }^{5} \chi 2=15.45$, d.f. $=1, p<0.001$ for C-allele carriers frequency in runners vs. swimmers

${ }^{6} \chi 2=18.43$, d.f. $=1, p<0.001$ for allele frequency in in runners vs. swimmers

${ }^{7} \chi 2=21.65, d . f .=4, p<0.001$ for genotype frequency in long distance swimmers vs. long distance runners and middle distance runners

${ }^{8} \chi 2=18.16$, d.f. $=2, p<0.001$ for C-allele carriers frequency in long distance swimmers vs. long distance runners and middle distance runners

${ }^{9} \chi 2=22.34$, d.f. $=2, p<0.001$ for allele frequency in long distance swimmers vs. long distance runners and middle distance runners

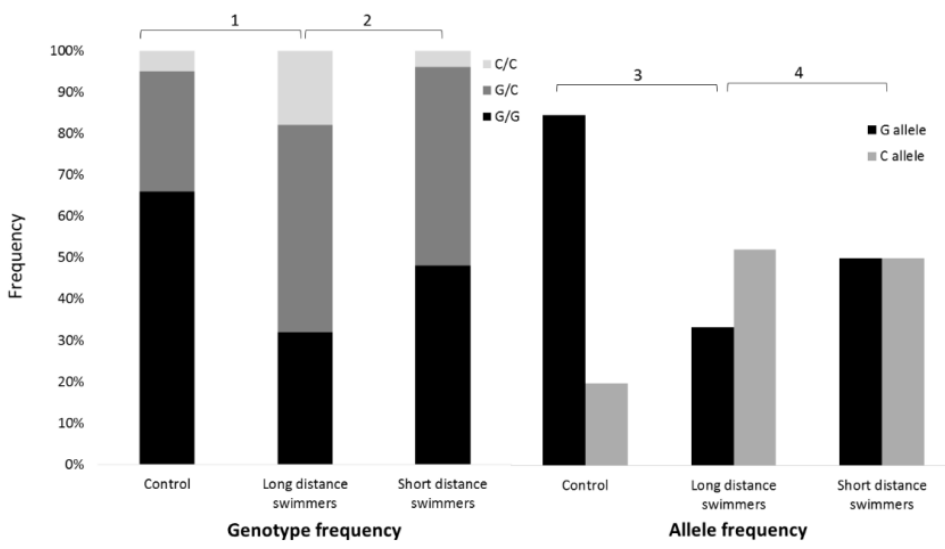

Figure 1

The IL-6 polymorphism (genotype and alleles frequencies) in swimmers and controls. ${ }^{1} \chi 2=7.56, d . f .=1, p<0.01$ for CC genotype frequency in controls vs. long distance swimmers.

${ }^{2} \chi 2=3.91, d$.f. $=1, p<0.05$ for CC genotype frequency in long distance swimmers vs. short distance swimmers

${ }^{3} \chi^{2}=18.24$, d.f. $=1, p<0.001$ for allele frequency in controls vs. long distance swimmers.

${ }^{4} \chi 2=4.26$, d.f. $=1, p<0.05$ for allele frequency in long distance swimmers vs. short distance swimmers 


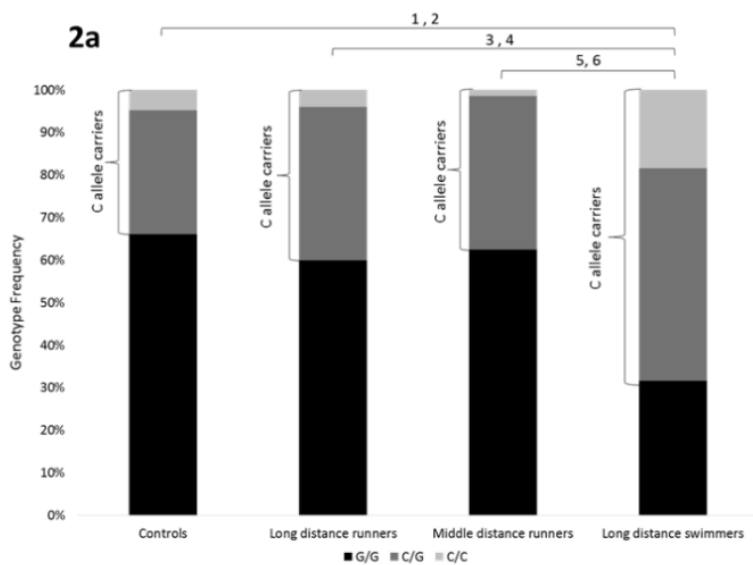

2b

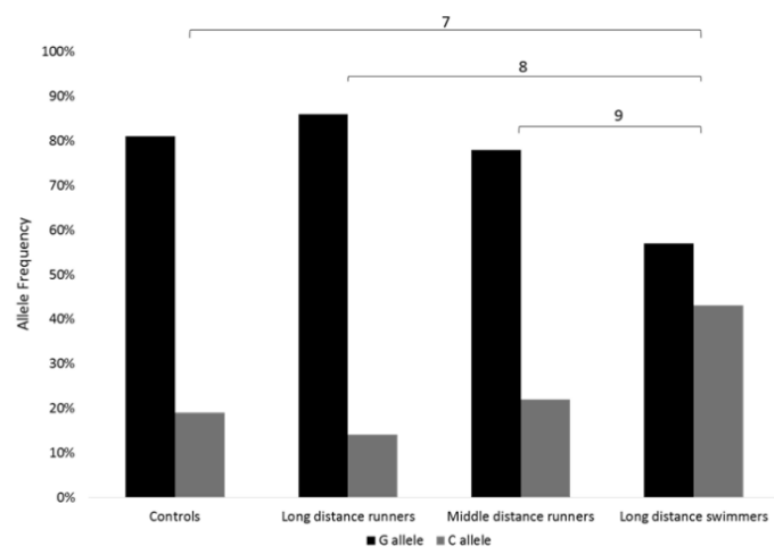

\section{Figure 2}

The IL-6 polymorphism ( $2 a$-genotype frequencies, $2 b$-allele frequencies) in endurance athletes (runners and swimmers)

${ }^{1} \chi 2=16.72$, d.f. $=2, p<0.001$ for genotype frequency in controls vs. long distance swimmers. ${ }^{2} \chi 2=7.56$, d.f. $=1, p<0.01$ for CC genotype frequency in controls vs. long distance swimmers. ${ }^{3} \chi^{2}=19.29$, d.f. $=2, p<0.001$ for genotype frequency in long distance runners $v$ s. long distance swimmers.

${ }^{4} \chi 2=6.79$, d.f. $=1, p<0.01$ for CC genotype frequency in long distance runners vs. long distance swimmers.

${ }^{5} \chi 2=9.05$, d.f. $=2, p<0.05$ for genotype frequency in middle distance runners $v$ s. long distance swimmers.

${ }^{6} \chi 2=4.89$, d.f. $=1, p<0.05$ for CC genotype frequency in middle distance runners vs. long distance swimmers.

${ }^{7} \chi 2=18.24$, d.f. $=1, p<0.001$ for allele frequency in controls vs. long distance swimmers.

${ }^{8} \chi 2=21.32, d . f .=1, p<0.001$ for allele frequency in long distance runners vs. long distance swimmers.

${ }^{9} \chi 2=9.22$, d.f. $=1, p<0.001$ for allele frequency in middle distance runners vs. long distance swimmers. 


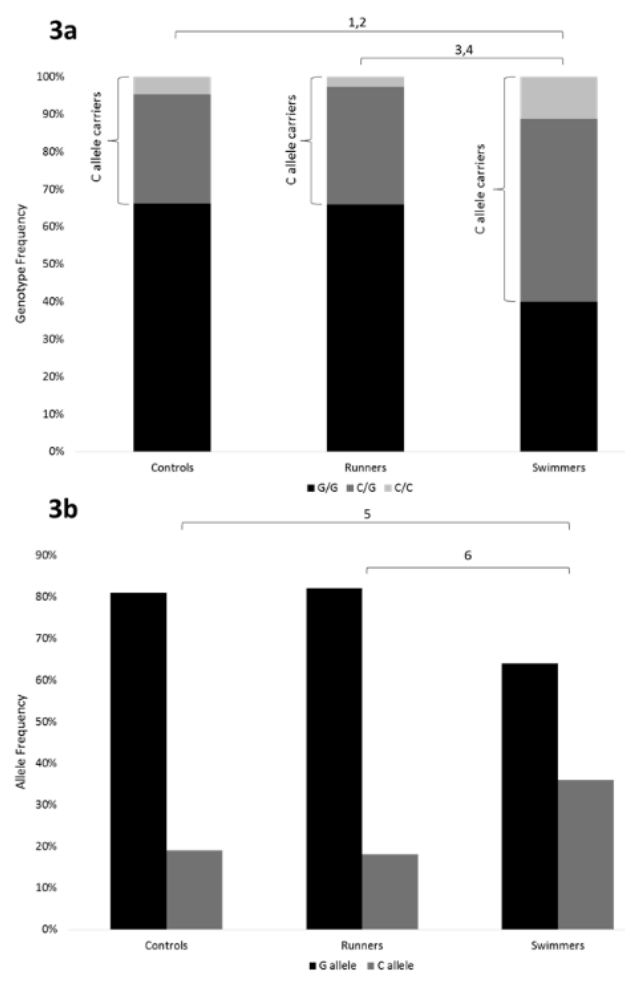

Figure 3.

The IL-6 polymorphism ( $3 a$-genotype frequencies, $3 b$-allele frequencies) in athletes and controls

${ }^{1} \chi 2=14.01$, d.f. $=2, p<0.001$ for genotype frequency in controls vs. swimmers.

${ }^{2} \chi 2=4.16, d . f .=1, p<0.05$ for CC genotype frequency in controls vs. swimmers.

${ }^{3} \chi 2=18.81$, d.f. $=2, p<0.001$ for genotype frequency in runners vs. swimmers.

${ }^{4} \chi 2=8.15, d . f .=1, p<0.001$ for CC genotype frequency in runners vs. swimmers.

${ }^{5} \chi 2=13.75, d . f .=1, p<0.001$ for allele frequency in controls vs. swimmers.

${ }^{6} \chi 2=18.43$, d.f. $=1, p<0.001$ for allele frequency in runners vs. swimmers.

The runners' genotype and allele frequencies did not differ significantly between the track and field subgroups (long, middle and short distance) and nor from the control group. However, the swimmers' genotype and allele frequencies differed significantly between the long and short-distance swimmers and from the controls. The long distance swimmers' CC genotype (18\%) and C allele frequency (43\%) were significantly higher compared to short distance swimmers (CC genotype: $4 \%, p<0.001$; C allele frequency: $29 \%, p<0.05)$ and compared to controls (CC genotype: 5\%, $p<0.001$; C allele frequency: 19\%, $p<0.001$ ) (Figure 1). Moreover, the CC genotype and $\mathrm{C}$ allele frequency were significantly higher in the long distance swimmers compared to the long distance runners ( 3 and $14 \%$ for the CC genotype and C allele frequency, respectively, $p<0.001$ ) and the middledistance runners (4 and 22\% for the CC genotype and $C$ allele frequency, respectively, $p<0.001$ ) (Figure 2).

Overall, the CC genotype and C allele frequency were significantly higher among the swimmers (11 and $36 \%$ for the CC genotype and $\mathrm{C}$ allele frequency, respectively) compared to the 
runners ( 3 and $14 \%$ for the CC genotype and C allele frequency, respectively) $(p<0.001)$; and controls (5 and 19\% for the CC genotype and C allele frequency, respectively) $(p<0.001)$ (Figure $3)$.

\section{Discussion}

We studied the frequency of the IL-6 174G/C polymorphism among elite Israeli swimmers and track and field athletes. In contrast to our hypothesis, the CC genotype and $\mathrm{C}$ allele frequency were significantly higher in longdistance swimmers compared to the long- and middle-distance runners (Figure 2), and compared to controls. In addition, the CC genotype and C allele frequency were significantly higher in longdistance compared to short-distance swimmers (Figure 1).

Elite swimmers and runners perform power, sprint, endurance and resistance training of high volume and at high intensities on a regular basis. As a consequence, they may be subjected to exercise-induced muscle damage and rhabdomyolysis.

Exercise-induced rhabdomyolysis refers to skeletal muscle injury that leads to leakage of muscle cell content into the circulation, including mainly the muscle proteins myoglobin and creatine kinase (CK) (Knochel, 1982). Clinical features associated with exercise-induced muscle damage are often nonspecific and include loss of muscle strength, reduced range of motion, muscle swelling and delayed onset muscle soreness. Despite a better prognosis of exercise-induced muscle damage compared to other causes of rhabdomyolysis, in extreme cases exertional muscle damage can be associated with serious complications, such as acute renal failure, electrolyte imbalance like hyperkalemia, which may lead to cardiac arrhythmias and even death (Landau et al., 2012). This serious, potentially life-threatening condition can develop unexpectedly, even under supervised training conditions (Moeckel-Cole and Clarkson, 2009).

More than 25,000 cases of exerciseassociated rhabdomyolysis are reported annually in the USA (Sauret et al., 2002), most of them among military recruits and long-distance runners (Kao et al., 1998; Kuipers, 1994; Nielsen and Mazzone, 1999; Schiff et al., 1978; Tietjen and Guzzi, 1989), and only a few among swimmers
(Galvez et al., 2008; Stella and Shariff, 2012). Eccentric contractions produced by muscle tension in activities like downhill running and jumping are more likely to cause muscle damage than concentric contractions. It was suggested that the reduced tendency of exercise-induces rhabdomyolysis in swimming activities is related, at least partially, to the fact that swimming involves predominantly concentric contractions. In addition, reduced weight bearing during water exercise and lower water temperature were also suggested as possible causes for the rarity of exertional muscle damage among swimmers (Galvez et al., 2008; Stella and Shariff, 2012).

Interestingly, there is wide interindividual variability in the degree of skeletal muscle damage and serum CK levels following strenuous eccentric exercise. This variability does not seem to be dependent on the athlete's age, race, body composition, and nor on the levels of physical activity, inactivity or fitness. It was suggested that genetic factors might play an important role in the development of exerciseinduced muscle damage and rhabdomyolysis (Landau et al., 2012). Specifically, an association was found between the IL-6 polymorphism and eccentric contraction-related muscle damage (Funghetto et al., 2013; Yamin et al., 2008).

The IL-6 response to exercise depends on the type of polymorphism $(-174 \mathrm{G} / \mathrm{C})$ within the IL-6 promoter (G-allele or C-allele) (Pedersen et al., 2003). Presence of the $G$ allele was associated with a higher circulating level of IL-6 (Fishman et al., 1998). The increase of IL-6 activity reduces muscle inflammation by stimulating the production of anti-inflammatory cytokines including IL-1ra, IL-10 (Petersen and Pedersen, 2005) and by inhibiting the production of proinflammatory cytokines such as TNF- $\alpha$ and IL-1 $\beta$ (Aderka et al., 1989), what results in a reduced post-exercise circulating CK level (Hirose et al., 2004). Increased production of IL-6 by skeletal muscles following acute exercise can also improve glucose utilization and sustain muscle energy demands by enhancing adenosine monophosphate-activated protein kinase activity (Carey et al., 2006). Thus, it seems that the protective effects of the IL-6 -174G genotype against exercise-induced muscle damage are due to increased muscle glucose uptake and restricted inflammation by stimulation of anti-inflammatory 
and inhibition of inflammatory cytokines' production, all known to play an important role in post-exercise muscle damage repair. In contrast, it was shown that individuals with one or more of the IL-6 -174C alleles had a higher post-exercise CK peak compared with individuals homozygous for the G allele (Yamin et al., 2008). The IL-6 174CC genotype was associated with a greater than threefold risk of massive CK response.

Based on previous reports of the relatively reduced tendency to develop exercise-induced rhabdomyolysis following swimming and the protective effect of the IL-6 -174G allele, we were surprised by the significantly higher CC genotype and $\mathrm{C}$ allele among long-distance swimmers compared to long and middle-distance runners and controls. This may suggest two important possibilities. First, despite the higher frequency of the $\mathrm{C}$ allele and CC genotype, exercise-associated rhabdomyolysis is less common in swimmers suggesting other swimming-related protective mechanisms such as reduced weight bearing in water sports, lower water temperature and predominant concentric-type exercise. Second, this finding may have implications for geneticallydependent sports selection. Both long distance runners and swimmers must have excellent endurance capabilities. Individuals carrying the IL-6 -174C allele are increasingly susceptible to develop eccentric exercise-induced muscle damage, in particular when performing exercises on the ground and therefore, their ability to recover from intense training might be reduced. Thus, it is possible that individuals with enhanced endurance characteristics, but with an unfavorable IL-6 -174C polymorphism subconsciously choose to specialize in swimming, a sport that would be significantly less affected by their genetic disadvantage.

Interestingly, long-distance swimmers also carried a significantly higher IL-6 CC genotype and $\mathrm{C}$ allele compared to short-distance swimmers. In addition, there were no significant differences in the frequency of IL-6 CC genotype and $C$ allele carriers between the short-distance swimmers and short-distance runners. This suggests that the possible genetically-dependent sports selection occurs mainly among endurance swimmers and not among sprinters.

A limitation of the present study is the relatively low number of participants for a genetic study. However, it should be noted that these athletes (runners and swimmers) are ranked among the nation's all-time record holders and best performances, and thus compose a very unique nationally elite population.

Talent identification in sport is a complicated task. During a natural selection process, the development of a gifted athlete is slow and often results in incorrect sport selections. As a consequence, many young athletes may compete in sport events that are not optimal for their inherent physical and physiological capacities. Predicting future success of young athletes is very challenging, since in addition to their genetic background, numerous additional biological, physical, physiological, environmental, and psychological factors may be involved in the process (Macarthur and North, 2005). Possible inherited traits that might be considered for talent identification include obvious features of body size, proportionality and onset of maturity, as well as muscle fiber type characteristics (endurance or power). Previous studies have described profiles of genetic polymorphisms that promote athletic excellence in endurance (Ben-Zaken et al., 2013; Ruiz et al., 2009; Williams and Folland, 2008) and power sports (Ben-Zaken et al., 2013; Ruiz et al., 2010). In the present study, we describe a genetic polymorphism that is possibly associated with sports selection based on the athlete's ability to effectively tolerate training. Overall, it is suggested that the use of genetic polymorphisms may serve as an additional assisting tool for talent identification and sports selection, and perhaps also for building effective training programs for youth athletes.

\section{References}

Aderka D, Le JM, Vilcek J. IL-6 Inhibits Lipopolysaccharide-Induced Tumor Necrosis Factor Production in Cultured Human Monocytes, U937 Cells, and in Mice. J Immunol (Baltimore, Md. : 1950), 1989; 143(11): 
$3517-3523$

Bennermo M, Held C, Stemme S, Ericsson CG, Silveira A, Green F, Tornvall P. 2004. Genetic Predisposition of the Interleukin-6 Response to Inflammation: Implications for a Variety of Major Diseases? Clin Chem, 2004; 50(11): 2136-2140

Ben-Zaken S, Meckel Y, Nemet D, Eliakim A. Genetic Score of Power-Speed and Endurance Track and Field Athletes. Scand J MedSci Spor, 2015; 25(2): 166-174

Bloomfield J, Blanksby BA, Ackland TR, Elliott BC. The Anatomical and Physiological Characteristics of PreAdolescent Swimmers, Tennis Players and Non-Competitors. Aust J Sci Med Sport, 1985; 17(3): 19-23

Carey AL, Steinberg GR, Macaulay SL, Thomas WG, Holmes AG, Ramm G, Prelovsek O, Hohnen-Behrens C, Watt MJ, James DE, Kemp BE, Pedersen BK, Febbraio MA. Interleukin-6 Increases InsulinStimulated Glucose Disposal in Humans and Glucose Uptake and Fatty Acid Oxidation in Vitro via AMP-Activated Protein Kinase. Diabetes, 2006; 55(10): 2688-2697

Eynon N, Ruiz JR, Meckel Y, Santiago C, Fiuza-Luces C, Gómez-Gallego F, Oliveira J, Lucia A. Is the -174 C/G Polymorphism of the IL6 Gene Associated with Elite Power Performance? A Replication Study with Two Different Caucasian Cohorts. Exp Physiol, 2011; 96(2): 156-162

Fishman D, Faulds G, Jeffery R, Mohamed-Ali V, Yudkin JS, Humphries S, Woo P. The Effect of Novel Polymorphisms in the Interleukin-6 (IL-6) Gene on IL-6 Transcription and Plasma IL-6 Levels, and an Association with Systemic-Onset Juvenile Chronic Arthritis. J Clin Invest, 1998; 102(7): 1369-1376

Funghetto SS, Prestes J, Silva Ade O, Farias DL, Teixeira TG, Vieira DC, Souza VC, Sousa NM, Navalta JW, Melo GF, Karnikowski MG. Interleukin-6 -174G/C Gene Polymorphism Affects Muscle Damage Response to Acute Eccentric Resistance Exercise in Elderly Obese Women. Exp Gerontol, 2013; 48(11): 1255-1259

Galvez R, Stacy J, Howley A. Exertional Rhabdomyolysis in Seven Division-1 Swimming Athletes. Clin J Sport Med, 2008; 18(4): 366-368

Gonçalves C EB, Rama L ML, Figueiredo AB. Talent Identification and Specialization in Sport: An Overview of Some Unanswered Questions. Int J Sport Ohysiol Perform, 2012; 7(4): 390-393

Hirano T. Interleukin 6 and Its Receptor: Ten Years Later. Int Rev Immunol, 1998; 16(3-4): 249-284

Hirose L, Nosaka K, Newton M, Laveder A, Kano M, Peake J, Suzuki K. Changes in Inflammatory Mediators Following Eccentric Exercise of the Elbow Flexors. Exerc Immunol Rev, 2004; 10: 75-90

Jeunemaitre X, Soubrier F, Kotelevtsev YV, Lifton RP, Williams CS, Charru A, Hunt SC, Hopkins PN, Williams RR, Lalouel JM, Corvol P. Molecular Basis of Human Hypertension: Role of Angiotensinogen. Cell, 1992; 71(1): 169-180

Kao PF, Tzen KY, Chen JY, Lin KJ, Tsai MF, Yen TC. Rectus Abdominis Rhabdomyolysis after Sit Ups: Unexpected Detection by Bone Scan. Brit J Sport Med, 1998; 32(3): 253-254

Knochel JP. Rhabdomyolysis and Myoglobinuria. Annu Rev Med, 1982; 33: 435-443

Kuipers H. Exercise-Induced Muscle Damage. Int J Sports Med, 1994; 15(3): 132-135

Landau ME, Kenney K, Deuster P, Campbell W. Exertional Rhabdomyolysis: A Clinical Review with a Focus on Genetic Influences. J Clinic Neuromuscul Dis, 2012; 13(3): 122-136

Macarthur DG, North KN. Genes and Human Elite Athletic Performance. Hum Genet, 2005; 116(5): 331-339

Miller SA, Dykes DD, Polesky HF. A Simple Salting out Procedure for Extracting DNA from Human Nucleated Cells. Nucleic Acids Res, 1988; 16(3): 1215.

Moeckel-Cole SA, and Clarkson PM. Rhabdomyolysis in a Collegiate Football Player. J Strength Cond Res, 2009; 23(4): 1055-1059

Nielsen C, Mazzone P. Muscle Pain after Exercise. Lancet, 1999; 353(9158): 1062

Pedersen BK, Steensberg A, Fischer C, Keller C, Keller P, Plomgaard P, Febbraio M, Saltin B. Searching for the Exercise Factor: Is IL-6 a Candidate? J Muscle Res Cell M, 2003; 24(2-3): 113-119 
Pedersen BK, Febbraio MA. Muscle as an Endocrine Organ: Focus on Muscle-Derived Interleukin-6. Physiol Rev, 2008; 88(4): 1379-1406

Petersen AMW, Pedersen BK. The Anti-Inflammatory Effect of Exercise. J Appl Physiol, 2005; 98(4): 1154-1162

Ruiz JR, Gómez-Gallego F, Santiago C, González-Freire M, Verde Z, Foster C, Lucia A. Is There an Optimum Endurance Polygenic Profile? J Physiol, 2009; 587(Pt 7): 1527-1534

Ruiz JR1, Arteta D, Buxens A, Artieda M, Gómez-Gallego F, Santiago C, Yvert T, Morán M, Lucia A. Can We Identify a Power-Oriented Polygenic Profile? J Appl Physiol, 2010; 108(3): 561-566

Ruiz JR, Buxens A, Artieda M, Arteta D, Santiago C, Rodríguez-Romo G, Lao JI, Gómez-Gallego F, Lucia A. The -174 G/C Polymorphism of the IL6 Gene Is Associaed with Elite Power Performance. J Science Med Sport, 2010; 13(5): 549-553

Sauret JM, Marinides G, Wang GK. Rhabdomyolysis. Am Fam Physician, 2002; 65(5): 907-912

Schiff HBE, MacSearraigh T, Kallmeyer JC. 1Myoglobinuria, Rhabdomyolysis and Marathon Running. Q J Med, 1978; 47(188): 463-472

Stella JJ, Shariff AH. Rhabdomyolysis in a Recreational Swimmer. Singapore Med J, 2012; 53(2): e42-44

Terry CF, Loukaci V, Green FR. Cooperative Influence of Genetic Polymorphisms on Interleukin 6 Transcriptional Regulation. J Biol Chem, 2000; 275(24): 18138-41814

Tietjen DP, Guzzi LM. Exertional Rhabdomyolysis and Acute Renal Failure Following the Army Physical Fitness Test. Mil Med, 1989; 154(1): 23-25.

Valovich McLeod TC, Decoster LC, Loud KJ, Micheli LJ, Parker JT, Sandrey MA, White C. National Athletic Trainers' Association Position Statement: Prevention of Pediatric Overuse Injuries. J Athl Train, 2011; 46(2): 206-220

Williams AG, Folland JP. Similarity of Polygenic Profiles Limits the Potential for Elite Human Physical Performance. J Physiol, 2008; 586(1): 113-121

Yamin C, Duarte JA, Oliveira JM, Amir O, Sagiv M, Eynon N, Sagiv M, Amir RE. IL6 (-174) and TNFA (-308) Promoter Polymorphisms Are Associated with Systemic Creatine Kinase Response to Eccentric Exercise. Eur J Appl Physiol, 2008; 104(3): 579-586

\section{Corresponding author:}

\section{Sigal Ben-Zaken,}

The Zinman College of Physical Education and Sports Sciences at the Wingate Institute, Netanya 42902, Israel.

Phone: 00972-9-8639257

Fax 00972-9-8639351

E-mail: sigalbz@wincol.ac.il 